\section{Christoph Bachmann}

\section{Gesundheit und Krankheit nach Paracelsus}

Gemäss dem Arzt, Alchemisten und Philosophen Theophrastus Bombastus von Hohenheim, besser bekannt als Paracelsus, entstehen Krankheiten, wenn das Gleichgewicht von Merkur (Lebenskraft, Geist), Sulfur (individualisierende Kraft, Seele) und Sal (formende, strukturierende Kraft, Körper) ins Wanken, d.h. ins Ungleichgewicht, gerät. Denn: Diese drei grundlegenden Prinzipien wirken in jedem Lebewesen und befinden sich im Gleichgewicht, solange dieses Lebewesen gesund ist. Jedes dieser drei Prinzipien zeigt seine Wirksamkeit auf einer bestimmten Ebene und in gewissen Substanzen.

\section{Merkur, Sulfur und Sal in der Pflanzenwelt}

Auch Pflanzen sind Lebewesen und tragen daher die drei Prinzipien Merkur, Sulfur und Sal in sich. Merkur wird in der Pflanzenwelt vom Alkohol dargestellt (Aqua vitae). Sulfur gibt den Pflanzen Farben, Formen und Geruch und wird durch ätherische Öle individualisiert. Sal ist die Struktur, der Pflanzenkörper. Dieses Prinzip wird in den Pflanzen durch die Mineralsalze repräsentiert (Kasten 1).

\section{Spagyrik}

Die Spagyrik ist ein ganzheitliches Naturheilverfahren, das durch ein spezielles Verfahren die Essenz einer Heilpflanze, das eigentliche Wirkprinzip, freisetzt und in eine anwend-

\title{
SAL-Essenzen - eine Erweiterung der Spagyrik: 12 verschiedene Kombinationen bei vielfältigen Beschwerden
}

Im folgenden Beitrag wird eine neue Art von spagyrischen Arzneimitteln, die sogenannten SAL-Komplexe, beschrieben. Die 12 Mittel orientieren sich jeweils an einem bestimmten Beschwerdebild. Dazu wurden jeweils 7 sich ergänzende Pflanzen zu einem umfassenden Komplex vereint. Die Besonderheit, dass beim spagyrischen Herstellungsprozess die Mineralsalze aus den Pflanzen wesentlich in diese Überlegungen mit einbezogen wurden, machen diese Produkte möglicherweise zu einer interessanten Ergänzung oder Alternative zur Biochemie nach Dr. Schüssler.

bare Form bringt. Der Ausdruck Spagyrik ist ein Zusammenschluss der beiden griechischen Wörter "spao», ich trenne, und «ageiro», ich füge zusammen.

Im Gegensatz zur herkömmlichen Chemie trennt die Spagyrik Substanzen nicht nach ihren materiellen Eigenschaften auf, sondern gliedert diese nach inhaltlichen Kriterien in sogenannte Prinzipien. Dabei können Substanzen, deren chemische Zusammensetzungen nichts miteinander $\mathrm{zu}$ tun haben, zu denselben alchemistischen Prinzipien gehören. Bei der Herstellung von spagyrischen Lösungen werden die drei Prinzipien von Paracelsus, d.h. Merkur, Sulfur und Sal, aus einer Pflanze erschlossen.

Es gibt verschiedene spagyrische Herstellungsverfahren. Am bekanntesten ist diejenige nach Zimpel. Die Firma Spagyros, ein Schweizer Hersteller spagyrischer Essenzen aus dem Schweizer Jura, hat ein eigenes, von Swissmedic anerkanntes Verfahren entwickelt. Darin hat sie gewisse Verfahrenstechniken gegenüber der Herstellungsmethode nach Zimpel weiter verfeinert. So orientieren sich die Gärzeiten nicht strikt nach einer bestimmten Vorschrift, sondern an der jeweiligen Pflanze. Sie kann von ca. 3 Wochen bis 3-4 Monaten dauern. Zudem wird die Maische nach dem Abpressen der festen Bestandteile schonend unter Vakuum destilliert. So werden die besonders empfindlichen Stoffe, die bei der sonst üblichen Wasserdampf-Destillation verloren gehen können, sorgfältig aufgefangen. Die festen Rückstände der Pflanze werden getrocknet, verbrannt und bei Temperaturen von rund 500 Grad kalziniert. Am Ende dieses Prozesses liegen mit der Asche die reinen Mineralsalze aus der Pflanze vor. Zum Abschluss wird diese Asche in der wässrigen Fraktion des Destillats aufgelöst, danach mit dem alkoholischen Teil vereint und später filtriert. Auf diese Weise entstehen sehr wohlriechende Essenzen.

\section{Biochemie nach Dr. Schüssler}

Der homöopathische Arzt Wilhelm Heinrich Schüssler veröffentlichte 1873 den Artikel «Eine abgekürzte Therapie gegründet auf Histologie und Cellularpathologie» und stellte darin eine neue Heilmethode, «die Biochemische Heilweise», vor. Schüssler ging von der Annahme aus,

\section{KARGER}

Fax +497614520714 Information@Karger.com www.karger.com
(๑) 2014 S. Karger GmbH, Freiburg 
dass fast alle Krankheiten einen gestörten Mineralhaushalt als Grundlage haben. Wenn z.B. ein bestimmter Mineralstoff fehlt, dann kann durch Zufuhr einer homöopathischen Potenz dieser Mineralstoff direkt in die Zelle gelangen und so die Krankheit heilen. Im Gegensatz zur Klassischen Homöopathie, die mit etwa 1000 verschiedenen Substanzen arbeitet, vertrat Schüssler die Meinung, 12 verschiedene Mineralsalze seien für die Behandlung von Krankheiten ausreichend. So entstanden die sogenannten Schüssler-Salze, die später auf 24 erweitert wurden. Die Schüssler-Salze werden auf chemische Weise synthetisiert oder isoliert und dann in die entsprechenden Potenzen potenziert; sie werden in den homöopathischen Potenzen D3, D6 oder D12 verabreicht.

\section{SAL-Essenzen}

In den SAL-Essenzen liegen diese Mineralsalze - durch den spagyrischen Prozess aus Heilpflanzen aufbereitet - vor. Dadurch werden die einzelnen Salze auch nicht isoliert, sondern sind in ihrem natürlichen
Gleichgewicht vorhanden. Aus Pflanzen gewonnene Mineralstoffe wurden durch den pflanzlichen Lebensprozess assimiliert und $\mathrm{zu}$ «Pflanzenstoff» umgebaut. Durch diese «Vegetabilisierung» wird das Wirkpotenzial erhöht, und die Mineralien sind in eine Form gebracht, die vom Körper
Tab. 1. Die 12 SALKomplexe.

\begin{tabular}{rll}
\hline Nr. & Name & Anwendungsgebiet \\
\hline 1 & Ruta graveolens comp. & Elastizität \\
2 & Avena sativa comp. & Aufbau \\
3 & Urtica dioica comp. & Sauerstoff und Licht \\
4 & Juglans regia comp. & Säftefluss und Entzündung \\
5 & Angelica archangelica comp. & Nervennahrung \\
6 & Allium sativum comp. & Zellstoffwechsel \\
7 & Melissa officinalis comp. & Entspannung \\
8 & Asparagus officinalis comp. & Wasserhaushalt \\
9 & Colchicum autumnale comp. & Säurehaushalt \\
10 & Levisticum officinale comp. & Entschlackung \\
11 & Polygonum aviculare comp. & Strukturierung, Haut und Gewebe \\
12 & Viola tricoloris comp. & Grundregulation \\
\hline
\end{tabular}

Kasten 1. Mineralsalze aus Pflanzen - ein interessanter Denkansatz: Kommentar

Die Spagyrik nicht «neu zu erfinden», jedoch auf eine neue Art nutzbar zu machen, war der Ansatz des Teams um Patrick Baumann, als es die SAL-Komplexe entwickelte. Ob diese Idee gelungen ist, wird der künftige Erfolg zeigen. Aus verschiedenen Überlegungen sind aber einige Aspekte der SAL-Komplexe bei näherer Betrachtung sehr spannend und verdienen unsere Aufmerksamkeit:

Es ist unbestritten, dass Pflanzen die benötigten Mineralstoffe aus dem ihnen zur Verfügung stehenden Boden aufnehmen. Dieses Phänomen macht man sich beispielsweise auch in der Anthroposophie zunutze, legt Metalle in die Erde ein und geht davon aus, dass diese in einer neuen, vegetabilisierten Form später für den Körper besser aufgenommen werden können.

Heute weiss man, dass der Körper anorganische Mineralien praktisch nicht aufnehmen kann. Sie werden als freie Stoffe im Blut transportiert und entweder ungenutzt über die Nieren wieder ausgeschieden oder aber in den Blutgefässen oder im Bindegewebe eingelagert. Anstelle anorganischer Mineralien benötigt der Mensch vielmehr organische, z.B. an Aminosäuren gebundene Mineralien, damit er diese in den Stoffwechsel einbauen kann. Die Bioverfügbarkeit ist ein in der Pharmakokinetik wichtiger Begriff und bezeichnet die Menge eines aufgenommenen Stoffes, der vom Körper auch tatsächlich für den Stoffwechsel genutzt werden kann. Die pharmazeutische Industrie nutzt diese Erkenntnis schon seit Langem und bindet daher Mineralstoffe, die in einem Medikament wirken sollen, an organische Stoffe wie Citrat, Gluconat oder Orotat.

Bei der spagyrischen Herstellung liegen die Mineralien bereits, wie oben beschrieben, von den Pflanzen assimiliert vor. Bei der Aufarbeitung werden sie durch Veraschung zwar wieder zu Oxiden. Beim darauf folgenden Auflösen entstehen aus den Oxiden jedoch basische, lösliche Hydroxide, die in der Tinktur zusammen mit den organischen Bestandteilen des spagyrischen Destillats vorliegen und so gemeinsam vom Körper aufgenommen werden können.

Wenn man in der Biochemie davon ausgeht, dass die Mineralsalze durch die homöopathische Verdünnung und Dynamisierung gut vom Körper aufgenommen werden, ist die «brückenfreie» Aufnahme von Mineralsalzen aus Pflanzen auf jeden Fall sehr naheliegend.

Aus einem weiteren Grund verdienen diese SAL-Komplexe unsere Aufmerksamkeit: Es wird immer wieder gesagt, in der pharmazeutischen Industrie seien nur noch die ganz grossen Firmen in der Lage, Neuheiten auf den Markt zu bringen. Dies mag für neue synthetische Arzneimittel durchaus stimmen, denn der Aufwand, ein neues synthetisches Arzneimittel zu entwickeln und dieses nach all den erforderlichen Prüfungen auch noch bei den Behörden registrieren zu können, ist heute so gewaltig, dass dafür Millionenbeträge investiert werden müssen, die für kleinere Firmen ausserhalb jeder Reichweite liegen. Dass aber im Bereich der Arzneimittel auch heute noch Nischen bestehen, die von kleineren Firmen belegt werden können, hat die Firma Spagyros mit den SAL-Komplexen gezeigt. Die in Gümligen ansässige Spagyros AG, ein kleines, komplementärmedizinisch engagiertes Team, hat sich ihre Erfahrungen mit der Spagyrik zunutze gemacht und diese SAL-Komplexe entwickelt. Sehr bemerkenswert ist dabei auch die Tatsache, dass diese aus dem Dialog zwischen Naturwissenschaftlern, Spagyrikern und Heilpraktikern entstanden sind - eine bemerkenswerte Zusammenarbeit! Diese könnte, würde die etablierte Schulmedizin endlich diesen Dialog aufnehmen, der Medizin ungeahnte Fortschritte bringen!

Es muss darauf hingewiesen werden, dass es sich bei den SAL-Komplexen um eine Novität handelt, die aus der spagyrischen und der biochemischen Tradition heraus, verbunden mit Überlegungen des Teams, das die SAL-Komplexe entwickelt hat, entstanden ist. Daher können die heute in einem wissenschaftlichen Artikel geforderten und oft im Übermass gelieferten Literaturreferenzen in diesem Beitrag nicht aufgeführt werden. Dies soll die Qualität der geleisteten Arbeit allerdings nicht schmälern! 


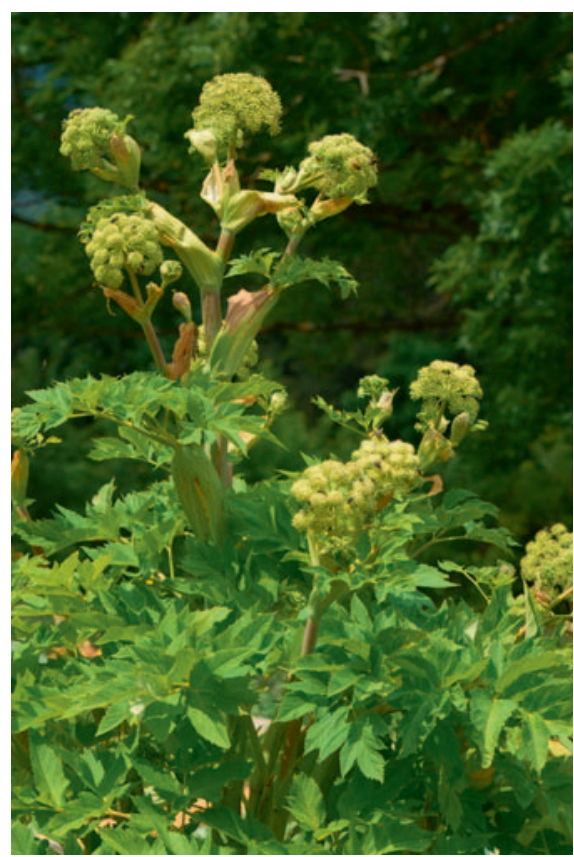

Abb. 1. Angelica archangelica.

leichter aufgenommen werden kann und die besser bioverfügbar ist.

SAL-Essenzen heissen so, weil bei der Konzeption der Mittel das Hauptaugenmerk auf dem spagyrischen Prinzip Sal lag. Aber natürlich sind wie bei allen Spagyrika auch die anderen Prinzipien, d.h. Sulfur und Merkur, in das Produkt eingearbeitet und harmonisieren dieses zu einer Ganzheit.

\section{Herstellung von SAL-Essenzen}

SAL-Essenzen werden gemäss der oben beschriebenen Methode von $\mathrm{Pa}$ trick Baumann, einem der Entwickler der SAL-Essenzen, hergestellt. Für diese Arzneimittel werden die jeweils 7 Pflanzen jedoch gemeinsam vergoren und durchlaufen den gesamten weiteren Prozess zusammen als eine Einheit. Es entsteht ein neues Ganzes, das mehr ist als die Summe seiner Einzelteile.

\section{SAL-Komplexe}

SAL-Komplexe sind also Mischungen aus 7 Pflanzen, die gemeinsam zu einer einzigen Essenz verarbeitet wurden. Jeder dieser Komplexe hat sein bestimmtes Anwendungsgebiet (Tab. 1), wie auch die beiden nachfolgenden Beispiele aufzeigen.

\section{SAL-Komplex Nr. 5: Angelica archangelica comp.}

\section{Zusammensetzung}

- Angelica archangelica (Engelwurz) (Abb. 1)

- Avena sativa (Hafer)

- Atropa belladonna (Tollkirsche)

- Eleutherococcus senticosus (Taigawurzel)

- Euphrasia officinalis (Augentrost)

- Galium odoratum (Waldmeister)

- Nicotiana tabacum (Tabak)

\section{Zusammenwirken}

Aus spagyrischer Sicht kann man über diese zusammen zu einem Komplex verarbeiteten 7 Pflanzen Folgendes sagen: Durch die spezifische Herstellung kann die nervenstärkende Wirkung von Tabak ohne seine anderen, schädlichen Wirkungen genutzt werden. Die Erfahrungsmedizin spricht dem Waldmeister vitalisierende Kräfte zu. Dem Augentrost werden Wirkungen im Kopf zugesprochen, wo er auf die Lymphbahnen wirkt. Hafer und die Taigawurzel stärken die Widerstandskraft und Adaptationsfähigkeit des Körpers. Die Tollkirsche hat eine entspannende Wirkung. In diesem «Wirkchor» kann dann Engelwurz ihren aufrichtenden Impuls entfalten.

\section{Anwendungsgebiet}

Wie immer in der Spagyrik ergeben sich aus einer solchen Zusammensetzung nicht einzelne Indikationen, sondern ein Anwendungsgebiet, zu dem die behandelte Person genau befragt und verschiedene Symptome erfasst werden. Der SAL-Komplex Angelica archangelica comp. wird bei Personen angewendet, die physisch und psychisch kraftlos sind und das Gefühl haben, die gegenwärtigen $\mathrm{He}$ rausforderungen nicht meistern $\mathrm{zu}$ können. Angelica archangelica comp. vermittelt neues Gleichgewicht und Anpassungsfähigkeit. Die behandelte Person erhält Kraft, neue Zuversicht und Halt und wird fähig, einer aktuellen Situation entgegenzutreten und die Herausforderungen zu meistern.

SAL-Komplex Nr. 7: Melissa officinalis comp.

\section{Zusammensetzung}

- Angelica archangelica (Engelwurz)

- Coffea arabica (Kaffee)

- Crataegus (Weissdorn)

- Datura stramonium (Stechapfel)

- Leonurus cardiaca (Herzgespann)

- Melissa officinalis (Melisse)

- Valeriana officinalis (Baldrian)

\section{Zusammenwirken}

In diesem SAL-Komplex findet man klassische Arzneipflanzen der Entspannung und Beruhigung: Melisse, Kaffee und Baldrian. Zusätzlich unterstützen und beruhigen Herzgespann und Weissdorn das Herz als Körpermitte im konkreten und übertragenen Sinn. Der Stechapfel gilt als wichtiges Mittel zur Entkrampfung und Abgrenzung und hilft bei übermässigen Sinneseindrücken. Engelwurz ist die Pflanze des Ausgleichs und der Stärkung.

\section{Anwendungsgebiet}

Melissa officinalis comp. passt zu einem gestressten Patienten, dessen Nerven überbelastet sind, der vor lauter Aktivitäten keine Durchsicht mehr hat und dessen Muskulatur, weil er ständig unter Strom steht, verkrampft ist. Melissa officinalis comp. hilft ihm, Ruhe und Entspannung zu finden, den Tagesablauf in einen vernünftigen Rhythmus zu bringen und nur so viel anzupacken, wie er bewältigen kann.

\section{Fallbeispiel: Stress, Zuversicht}

Das folgende Fallbeispiel ist ein gestellter Fall, der zeigen soll, wie bei 
der Beratung und Anwendung von SAL-Komplexen vorgegangen werden kann:

Ein Mann, ca. 45 Jahre alt, wirkt nervös und erkundigt sich nach einem natürlichen Schlafmittel; sein Schlaf sei seit einigen Monaten schlecht. Er habe Mühe einzuschlafen, erwache jede Nacht mehrmals und fühle sich am Morgen nicht genügend ausgeruht.

Auf eine gezielte Nachfrage gibt er an, seit Längerem beruflich sehr unter Druck zu stehen. Er arbeitet in einer grösseren Firma und wurde vor einem Jahr befördert. Dies brachte ihm aber Mehrarbeit, für die er oft Überstunden machen muss. Seither ist sein All- tagsrhythmus durcheinander. Am Mittag nimmt er sich kaum Zeit, richtig zu essen, sondern isst vor dem Computer ein Sandwich. Am Abend, wenn er nach Hause kommt, findet er keine Ruhe. Oft spürt er eine innere Verkrampfung und kann sich nicht mehr richtig konzentrieren.

Da der Mann nicht unbedingt $\mathrm{Zu}-$ versicht ausstrahlt, geht eine behutsam gestellte Frage in diese Richtung. Erstaunlicherweise gesteht er nach einigem Zögern, dass er sich in seiner neuen Stellung oft überfordert fühlt und manchmal findet, das Ganze sei für ihn eine Nummer zu gross. Dies will er aber mit entsprechendem Einsatz wettmachen, da er seine Arbeit trotzdem liebt und ein hohes Pflichtbewusstsein besitzt.

Damit ist die Entscheidung für eine Empfehlung schnell getroffen: Dem Patienten wird empfohlen, die beiden SAL-Komplexe 5 und 7 anzuwenden.

Melissa officinalis comp. wirkt gegen die stressbedingte Nervosität, die Angespanntheit und die Schlafstörungen. Die zusätzliche Einnahme von Angelica archangelica comp. verleiht dem Mann Zuversicht und die Gewissheit, die auf ihn zukommenden Aufgaben bewältigen zu können.

Von beiden SAL-Komplexen zusammen wird 8-bis 10-mal täglich ein Sprühstoss eingenommen. 